\title{
Transcatheter aortic valve implantation in Asia
}

\author{
Jimmy Kim Fatt Hon, Edgar Tay \\ National University Heart Centre, National University of Singapore, Singapore \\ Correspondence to: Dr. Jimmy Kim Fatt Hon, MB ChB, FRCS (C-Th). Department of CTVS, National University Heart Centre, National University \\ of Singapore, 1E, Kent Ridge Road, NUHS Tower Block Level 9, Singapore 119228, Singapore. Email: jimmy_hon@nuhs.edu.sg.
}

\begin{abstract}
The use of transcatheter aortic valve implantation (TAVI) to treat severe symptomatic aortic valve stenosis has increased exponentially in the last decade. This rapid expansion was seen predominantly in Western developed nations and has been fuelled by favorable results reported from a plethora of well-publicized randomized controlled trials, large retrospective series and national registries. Now, TAVI has become the standard of care for inoperable patients and an alternative to open surgery in patients who are at intermediate to high risk for open surgery. Notwithstanding these positive results, Asia has been relatively slow to adopt this technology despite a potentially large patient pool. Unique features of Asian medical environments and differences in Asian anatomy affecting TAVI uptake in Asia will be discussed. This article serves to outline the various challenging aspects of disseminating TAVI in Asian countries.
\end{abstract}

Keywords: Transcatheter aortic valve implantation (TAVI); transcatheter aortic valve replacement (TAVR); aortic; transcatheter; Asia

Submitted Jul 24, 2017. Accepted for publication Aug 14, 2017.

doi: 10.21037/acs.2017.08.05

View this article at: http://dx.doi.org/10.21037/acs.2017.08.05

\section{Introduction}

Transcatheter aortic valve implantation (TAVI) has revolutionized the treatment of severe symptomatic aortic stenosis. The adoption of this technology to treat high risk and inoperable patients has increased exponentially over the last decade in the developed Western world. TAVI is now the standard of care for inoperable patients and an alternative to open surgery for patients who are at high risk of open surgery. Recently, TAVI has also been recognized to be a reasonable alternative treatment strategy for patients at medium operative risk who have severe symptomatic aortic stenosis (1). To date, more than 100,000 TAVI procedures have been performed worldwide, driven by positive results from numerous landmark randomized controlled trials and large national registries reporting good short to medium term survival and safety data (1-7). Despite this, the majority of these cases were done in Europe and the USA and the adoption of this technology in Asia remained relatively slow. Publications related to TAVI cases performed in Asia are also relatively few, when compared to those from Western countries.

\section{Asian case volume}

To date, only about 10,000 TAVI cases have been performed in Asia. Edwards Sapien (Edwards Lifescience, Irvine, CA, USA) family balloon expandable prosthesis is, by far, the most common TAVI prosthesis used in Asia. Edwards Sapien valve was the first TAVI device to be implanted in Asia back in 2009 in Singapore. Up until the end of 2016, about 6,000 TAVI procedures using the Edwards Sapien device were carried out in Asia. Most of these cases were performed in Japan (around 5,200 cases) despite the fact that the Sapien valve was only launched in Japan in 2013. This leaves only about 800 Edwards Sapien valves being implanted in the rest of Asia since 2009. To date, the Medtronic CoreValve (Medtronic Incorporation, Minneapolis, MN, USA) self-expanding prosthesis is the second most used TAVI valve in Asia, having been implanted in about 3,300 cases. The Boston Scientific Lotus valve (Boston Scientific Corporation, Marlborough, MA, USA) and Abbott (previously St. Jude Medical) Portico valve (Abbott, Chicago, Illinois, USA) were both launched in Asia in 2015. The Boston Scientific Lotus valve has since 
been implanted in 264 cases in Asia up until the end of 2016. Portico valve had their first case in Asia implanted in Hong Kong and have since performed about 36 implants in Asia, outside of Japan. Currently, there is a Portico Japan trial on-going for the purpose of product registration for commercialization in Japan but the total number of valves implanted there are not available for reporting. Symetis Acurate valve (Boston Scientific) entered the Japanese market in 2013 and currently also has an on-going trial for the same purpose.

Currently, about 800 TAVI cases have been carried out in China using at least six different valves, namely the Venus A-valve (Venus MedTech, Hangzhou, China), the J-Valve (Jie Cheng Medical Technologies, Suzhou, China), the MicroPort VitaFlow valve (MicroPort Medical, Shanghai, China), the Edwards Sapien XT valve, Medtronic CoreValve, and the Boston Scientific Lotus valve. The first three valves on this list are locally manufactured valves from China and the published short-term and midterm results of these valves have been highly encouraging $(8,9)$. However, there is currently only one valve that is fully commercialized in China. Venus A-valve has recently been approved by the China Food and Drug Administration for sale in China, making it the first approved TAVI device in China. So far, about 380 Venus A-valve TAVI implants have been performed in China.

Asia, being the largest and most populous continent in the world, houses an estimated population of 4.4 billion people. When compared to Europe's population of 741 million, the uptake of TAVI procedure in Asia could be considered to be extremely low. However, the life expectancies of some of the most populous countries in Asia (e.g., Indonesia and Philippines) are in their low 60's, hence, patients from these countries are much less likely to live long enough to develop degenerative aortic stenosis. Nevertheless, factors contributing to this slow adoption rate in Asia includes lack of government funding resulting in reimbursement challenges, high cost of TAVI devices, lack of screening and treatment infrastructure, lack of a Heart Team and structured training programme, and the presence of potentially challenging anatomical features.

\section{Reimbursement/funding and cost}

Unlike in most of Europe and USA where TAVI devices are government funded, most Asian countries' patients have to pay for and fund their valve themselves. The exception to this is Japan where TAVI devices are funded by the Japanese government. Little has been published in this area. Pricing is a sensitive sale strategy related information and understandably, manufacturers are not all open to disclosing exact pricing figures. Currently in Asia, commercially implanted TAVI devices from multinational manufacturers are priced between US $\$ 20,000$ to US $\$ 40,000$. The latest generation of the Edwards Sapien 3 (Edwards Lifescience) valve and the Medtronic Evolut R (Medtronic Incorporation) valve are currently at the top end of this price bracket, with the rest of the multinational valve competitors occupying the middle and lower half of this price range. TAVI procedures have been found to be cost-effective in treating high risk and inoperable patients in many Western countries (10-12). However, these analyses are usually country-specific and dependent on local healthcare funding structure. For example, unpublished analysis of local treatment cost in Singapore revealed a relatively low cost for medical therapy for severe aortic stenosis and open surgery for aortic valve replacement, hence making cost-effectiveness of TAVI procedures to be less favorable compared to those published from Western countries, at the present price range in Asia. It was estimated that for TAVI to be cost-effective in Singapore, TAVI device price has to be in the region of US\$15,000. In the many developing countries in Asia, this figure will vary and it will be highly dependent on the cost of medical therapy and open surgery.

\section{Registry and trials}

The largest TAVI registry currently in Asia is the Japan Transcatheter Aortic Valve Replacement (TAVR) registry. This registry contains data of all the TAVI cases that were carried out in Japan and has in excess of 8,000 cases, although no publication has emerged from this registry so far. Another large TAVI registry in Asia is the Japan OceanTAVI (Optimized Transcatheter Valvular Intervention) registry (13). This is an on-going, prospective, multicentre TAVI registry, collecting TAVI data from at least nine Japanese institutions. It has data from at least 2,500 cases reported since 2013. There are two other Asian prospective multicentre TAVI registries that are actively recruiting patients. The Asian Pacific TAVR Multicentre Registry (NCT02195102) enrolls patients spanning eight Asian countries from at least 10 institutions and currently has more than 1,000 patients in this database. The Asian TAVR is a Korean initiative with more than 840 patients recruited from 11 institutions spanning five Asian countries (14). 
These registries provide valuable information such as the type and size of devices used in Asia, patients' unique anatomical characteristics and procedure outcomes. Such information will be useful to improve TAVI outcomes in Asia and to develop device improvement beneficial to the Asian patients. Further strategies to strengthen these registries include the use of core lab for reporting of outcomes and making reporting mandatory.

There are relatively few TAVI trials conducted in Asia. The most obvious limitations in this aspect relate to the relatively low TAVI case volume seen in most Asian countries compared to European centres and also the lack of trial infrastructure in many developing nations.

Currently, there are no reported randomized controlled TAVI trials from Asia. By far, the majority of clinical trials were carried out in Japan, fuelled by the need for locally generated data for product registration and commercialization in Japan. The PREVAIL Japan trial was performed as a prospective, multicentre, non-randomized and pivotal clinical trial in Japan using the Edwards Lifescience balloon expandable Sapien XT (Edwards Lifescience) valve (15). The MDT-2111 Japan trial on the other hand, was a relatively similar trial that used the Medtronic self-expanding CoreValve (Medtronic) (16). Results from both these Japanese trials were pivotal for product registration and commercialization in Japan. Various multinational TAVI device manufacturers have since also entered the Japanese market, starting their own respective clinical trials and working towards full commercialization of their respective TAVI devices. Boston Scientific Lotus valve (REPRISE Japan trial, NCT02491255), Symetis Acurate valve, and Abbott Portico valve all have their respective on-going clinical trials in Japan for this purpose.

China also has the same requirement for locally generated data for device commercialization and hence, the equivalent trial for Venus-A valve in China was the Venus-A trial (NCT01683474) (17). The J-Valve (NCT03025971) and the MicroPort VitaFlow valve (NCT02221921) both have on-going clinical trials in China in preparation for product commercialization.

\section{Anatomy}

Asian patients have several unique anatomical characteristics. First, Iliofemoral vessel diameter is likely to be smaller due to smaller Asian physique. Chiam et al. documented several factors in predicting smaller iliofemoral vessel size (18). These include female sex, lower body surface area, and smoking history. Secondly, Asian populations generally have a smaller aortic valve annulus $(19,20)$. In addition, Watanabe et al. found that the sinus of Valsalva, sinus of Valsalva height, sinotubular junction, and sinotubular junction height were also smaller in Japanese patients when compared to Western patients, suggesting that these patients had a shallower sinus of Valsalva (19). Coronary ostium height was also found to be lower in this study. These findings increase the theoretical risk of aortic annulus rupture and coronary occlusion.

Bicuspid aortic valve has been reported to be very common in Chinese patients in China. The prevalence was reported to be near $50 \%$ in patients with severe aortic stenosis undergoing TAVI procedures (17). Further, in the same study, it was also found that there was three-fold excess of leaflet calcium burden in patients with trileaflet aortic valve, when compared to a Western population. These unique characteristics seen in Chinese patients pose specific challenges for TAVI procedures.

\section{Heart Team, infrastructure, and training program}

The concept of Heart Team management of heart valve disease has been well-published and well-established in Western countries. A typical valvular Heart Team consists of the cardiothoracic surgeon, the interventional cardiologist, a structural heart disease expert, and imaging specialists, amongst others. This Heart Team approach has been recommended by multiple specialty societies and is also mandated by the U.S. Food and Drug Administration and Centers for Medicare and Medicaid Services for regulatory and reimbursement purposes (21).

In Asia, most if not all TAVI centres have existing Heart Teams in place to manage valvular heart disease. Hence, TAVI is done mainly in hospitals with cardiac surgery cover. Similar to Western centers, the Heart Team approach to managing aortic stenosis patients is mandated by TAVI device manufacturers before new centres are allowed to carry out TAVI procedures. Although Heart Teams are fairly well developed in most hospitals in developed Asian nations, they are still fairly sporadic in developing countries where both intervention cardiology and cardiac surgery services are still under development and expansion. Informal discussion with industry partners revealed that many interested cardiologists and surgeons in Asia have sought out TAVI device manufacturers requesting to start TAVI programmes. However, various 
factors have prevented the initiation of new TAVI centres in many developing countries despite large patient volume. Cardiac surgeons and interventional cardiologists in many developing countries are busy due to large patient volume and are still working fairly independently of each other. The inability to form a cohesive and effective Heart Team has often been quoted as a factor in the slow initiation of a new TAVI centre, although this usually resolves itself when both parties are persuaded to understand the mandatory requirement of a well-functioning Heart Team to start a successful TAVI programme.

Other factors why TAVI programmes are harder to start in Asia include the lack of satisfactory infrastructure such as dedicated heart valve clinics and efficient echocardiogram services, hence limiting effective screening. Lack of efficient and rapid CT scan services will also limit screening of a large sustainable patient pool. Like in many Western nations, a hybrid operating room is desired and recommended for TAVI procedures in Asia although not mandatory. However, the absence of a good size catheterization lab will have a negative impact on the prospect of any centre starting a new TAVI programme.

Structured TAVI education programme and learning opportunities like those seen at large conferences in Europe and the USA are also less available to these countries. However, hospitals with a well-functioning Heart Team, good infrastructure, large annual aortic valve volume, and long-term sustainable funding structure will enjoy full structured training opportunities provided by the device manufacturers, to ensure safe and successful programmes. Much of this training will involve device manufacturers flying in experienced high-volume Western physician proctors for the initial cases in a new programme. However, the proctoring period tends to be longer due to the lowercase volume and infrequent cases. As a result, the upfront cost of starting a new TAVI centre in Asia is likely to be significantly higher. However, this higher upfront start-up cost for new Asian centers is mitigated by the fact that it is usually not necessary to conduct clinical trials in most Asian countries to commercialize TAVI devices. Japan and China are the exceptions to this rule.

\section{Outcomes}

Asian patients, with their unique challenging anatomical features described previously, pose safety concerns when performing TAVI procedures. Moreover, the learning curve is likely prolonged with the lower-case volume and infrequent cases in many Asian TAVI centers. However, these concerns were ablated with the publication of favorable results from various Asian clinical series $(15,16,22$ $25)$. We reported the mid-term results of our own modest series last year consisting of 59 patients who underwent TAVI procedure between 2010 and 2015 (22). Devices used in our series were 48 Edwards SAPIEN XT, two classic CoreValves, two current generation CoreValve Evolut R (Medtronic Incorporation), one Boston Scientific Lotus valve, one Engager valve (Medtronic Incorporation, $\mathrm{MN}$, USA), one SAPIEN 3 valve and two JenaValves (JenaValve Technology Gmbh, Munich, Germany). Logistic EuroSCORE in our series was $18.7 \%$. The 30 -day, 1 -year, and 2-year mortality rates were $6.8 \%, 14 \%$ and $20.9 \%$, respectively. The 30 -day stroke rate was $1.7 \%$. These are comparable results to other major Western registry data. Watanabe et al. (19) also compared directly the clinical outcomes of the Japan PREVAIL trial and a European single-center experience and found the Japanese results to be acceptable and as safe as those of a European singlecenter cohort.

Outcome data published from 2 large Asian registry also revealed very favorable results when compared to Western series. Thirty day mortality rate from the Japanese OCEAN-TAVI registry was reported as $1.9 \%$ as only 23 patients of the 1,215 patients died in this time period (13). Yoon et al. reported results of 848 Asian patients from 5 Asian countries. The 30-day mortality in this cohort was $2.5 \%$ and the 1 -year mortality was $10.8 \%$ (14).

Several reports have documented increased paravalvular leak rates and increased use of permanent pacemaker when TAVI was used to treat bicuspid aortic valve stenosis (26-28). Yousef et al. (27) and Mylotte et al. (26) separately reported the rates of paravalvular leak of $2+$ or greater as $30.8 \%$ and $28.4 \%$ respectively. TAVI used in Asian patients with bicuspid valve stenosis has yielded satisfactory outcomes when compared to Western patients $(29,30)$. China reported unusually high prevalence of bicuspid aortic valve in Chinese patients with severe aortic valve stenosis and these patients were associated with high leaflet calcium burden. Despite this challenging anatomy, TAVI procedures performed in these patients have also resulted in highly acceptable safety results, especially in relation to paravalvular leak rates and new pacemaker use (30). Liu et al. reported a series of 15 bicuspid aortic valve stenosis patients who underwent TAVI and no patient had paravalvular leak greater than $2+$ and only two out of 15 patients $(13.3 \%)$ needed new pacemaker implantation. With 
current new generation TAVI devices, especially those with an outer skirt covering the valve stent, such as the Edwards Sapien 3 valve and the Boston Scientific Lotus valve, TAVI paravalvular leak rate in bicuspid aortic valve stenosis is expected to decrease significantly $(28,31)$.

In addition to the use of TAVI in bicuspid aortic valve stenosis, various off-label use of TAVI device has also been reported by Asian centres. Case reports reported cases such as utilizing TAVI in severe symptomatic aortic regurgitation (32) and transcatheter valve-in-degenerated bioprosthetic valve implantation (33) which have been attempted successfully in Asia.

\section{Conclusions}

Despite the various challenges discussed that contributed to the relatively slow adoption of TAVI technology in Asia and the relatively lower number of cases performed in many Asian countries, results of TAVI procedures performed in Asia have been good and comparable to those from high-volume Western countries. The volume of TAVI procedures is definitely growing in Asia. Asian registries are also growing, improving and maturing. It is now timely to promote greater collaboration between even more Asian TAVI units to share clinical data and training programmes, and learn from one another at local and regional conferences, so as to advance our knowledge and understanding of performing TAVI in Asia. Furthermore, we would like to see multinational TAVI device manufacturers working even closer than ever with the various governments of developing Asian nations to develop a pricing strategy in Asia that will allow patients with severe aortic stenosis to benefit from a life-saving procedure, such as TAVI.

\section{Acknowledgements}

None.

\section{Footnote}

Conflicts of Interest: The authors have no conflicts of interest to declare.

\section{References}

1. Leon MB, Smith CR, Mack MJ, et al. Transcatheter or Surgical Aortic-Valve Replacement in Intermediate-Risk
Patients. N Engl J Med 2016;374:1609-20.

2. Daubert MA, Weissman NJ, Hahn RT, et al. Long-Term Valve Performance of TAVR and SAVR: A Report From the PARTNER I Trial. JACC Cardiovasc Imaging 2016. [Epub ahead of print].

3. Kapadia SR, Leon MB, Makkar RR, et al. 5-year outcomes of transcatheter aortic valve replacement compared with standard treatment for patients with inoperable aortic stenosis (PARTNER 1): a randomised controlled trial. Lancet 2015;385:2485-91.

4. Mack MJ, Leon MB, Smith CR, et al. 5-year outcomes of transcatheter aortic valve replacement or surgical aortic valve replacement for high surgical risk patients with aortic stenosis (PARTNER 1): a randomised controlled trial. Lancet 2015;385:2477-84.

5. Zahn R, Gerckens U, Grube E, et al. Transcatheter aortic valve implantation: first results from a multi-centre realworld registry. Eur Heart J 2011;32:198-204.

6. Walther T, Hamm CW, Schuler G, et al. Perioperative Results and Complications in 15,964 Transcatheter Aortic Valve Replacements: Prospective Data From the GARY Registry. J Am Coll Cardiol 2015;65:2173-80.

7. Auffret V, Lefevre T, Van Belle E, et al. Temporal Trends in Transcatheter Aortic Valve Replacement in France: FRANCE 2 to FRANCE TAVI. J Am Coll Cardiol 2017;70:42-55.

8. Luo X, Wang X, Li X, et al. Transapical transcatheter aortic valve implantation using the J-Valve system: A 1-year follow-up study. J Thorac Cardiovasc Surg 2017;154:46-55.

9. Liao YB, Zhao ZG, Wei X, et al. Transcatheter aortic valve implantation with the self-expandable venus $\mathrm{A}-\mathrm{Valve}$ and CoreValve devices: Preliminary Experiences in China. Catheter Cardiovasc Interv 2017;89:528-33.

10. Fairbairn TA, Meads DM, Hulme C, et al. The costeffectiveness of transcatheter aortic valve implantation versus surgical aortic valve replacement in patients with severe aortic stenosis at high operative risk. Heart 2013;99:914-20.

11. Indraratna $\mathrm{P}, \mathrm{Ang} \mathrm{SC}, \mathrm{Gada} \mathrm{H}$, et al. Systematic review of the cost-effectiveness of transcatheter aortic valve implantation. J Thorac Cardiovasc Surg 2014;148:509-14.

12. Orlando R, Pennant M, Rooney S, et al. Cost-effectiveness of transcatheter aortic valve implantation (TAVI) for aortic stenosis in patients who are high risk or contraindicated for surgery: a model-based economic evaluation. Health Technol Assess 2013;17:1-86.

13. Shimura T, Yamamoto M, Kano S, et al. Impact of the Clinical Frailty Scale on Outcomes After Transcatheter 
Aortic Valve Replacement. Circulation 2017;135:2013-24.

14. Yoon SH, Ahn JM, Hayashida K, et al. Clinical Outcomes Following Transcatheter Aortic Valve Replacement in Asian Population. JACC Cardiovasc Interv 2016;9:926-33.

15. Sawa Y, Takayama M, Mitsudo K, et al. Clinical efficacy of transcatheter aortic valve replacement for severe aortic stenosis in high-risk patients: the PREVAIL JAPAN trial. Surg Today 2015;45:34-43.

16. Sawa Y, Saito S, Kobayashi J, et al. First clinical trial of a self-expandable transcatheter heart valve in Japan in patients with symptomatic severe aortic stenosis. Circ J 2014;78:1083-90.

17. Jilaihawi H, Wu Y, Yang Y, et al. Morphological characteristics of severe aortic stenosis in China: imaging corelab observations from the first Chinese transcatheter aortic valve trial. Catheter Cardiovasc Interv 2015;85 Suppl 1:752-61.

18. Chiam PT, Koh AS, Ewe SH, et al. Iliofemoral anatomy among Asians: implications for transcatheter aortic valve implantation. Int J Cardiol 2013;167:1373-9.

19. Watanabe Y, Hayashida K, Takayama M, et al. First direct comparison of clinical outcomes between European and Asian cohorts in transcatheter aortic valve implantation: the Massy study group vs. the PREVAIL JAPAN trial. J Cardiol 2015;65:112-6.

20. Tay EL, Lew PS, Poh KK, et al. Demographics of severe valvular aortic stenosis in Singapore. Singapore Med J 2013;54:36-9.

21. Tommaso CL, Bolman RM 3rd, Feldman T, et al. Multisociety (AATS, ACCF, SCAI, and STS) expert consensus statement: operator and institutional requirements for transcatheter valve repair and replacement, part 1: transcatheter aortic valve replacement. J Am Coll Cardiol 2012;59:2028-42.

22. Chew N, Hon JK, Yip WL, et al. Mid-term study of transcatheter aortic valve implantation in an Asian population with severe aortic stenosis: two-year valve academic research consortium-2 outcomes. Singapore Med J 2017;58:543-50.

23. Tay EL, Yip JW, Teoh KL, et al. Transcatheter aortic

Cite this article as: Hon JK, Tay E. Transcatheter aortic valve implantation in Asia. Ann Cardiothorac Surg 2017;6(5):504509. doi: 10.21037/acs.2017.08.05 valve implantation: implications for Asian patients. Asian Cardiovasc Thorac Ann 2013;21:396-401.

24. Fuku Y, Goto T, Komiya T, et al. Thirty-day outcome of transcatheter aortic valve implantation with the edwards SAPIEN XT prosthesis via the transiliofemoral approach. Circ J 2014;78:1357-63.

25. Maeda K, Kuratani T, Torikai K, et al. New SelfExpanding Transcatheter Aortic Valve Device for Transfemoral Implantation- Early Results of the Firstin-Asia Implantation of the ACURATE Neo/TF(TM) System. Circ J 2015;79:1037-43.

26. Mylotte D, Lefevre T, Sondergaard L, et al. Transcatheter aortic valve replacement in bicuspid aortic valve disease. J Am Coll Cardiol 2014;64:2330-9.

27. Yousef A, Simard T, Webb J, et al. Transcatheter aortic valve implantation in patients with bicuspid aortic valve: A patient level multi-center analysis. Int J Cardiol 2015;189:282-8.

28. Perlman GY, Blanke P, Webb JG. Transcatheter aortic valve implantation in bicuspid aortic valve stenosis. EuroIntervention 2016;12:Y42-5.

29. Zhao ZG, Jilaihawi H, Feng Y, et al. Transcatheter aortic valve implantation in bicuspid anatomy. Nat Rev Cardiol 2015;12:123-8.

30. Liu XB, Jiang JB, Zhou QJ, et al. Evaluation of the safety and efficacy of transcatheter aortic valve implantation in patients with a severe stenotic bicuspid aortic valve in a Chinese population. J Zhejiang Univ Sci B 2015;16:208-14.

31. Yoon SH, Lefevre T, Ahn JM, et al. Transcatheter Aortic Valve Replacement With Early- and New-Generation Devices in Bicuspid Aortic Valve Stenosis. J Am Coll Cardiol 2016;68:1195-205.

32. Tay EL, Kong WK, Yip JW, et al. Successful Transcatheter Aortic Valve Replacement for Severe Aortic Valve Regurgitation Following a David I Valve-Sparing Procedure. JACC Cardiovasc Interv 2017;10:e101-e3.

33. Chiam PT, Ewe SH, Soon JL, et al. Percutaneous transcatheter aortic valve implantation for degenerated surgical bioprostheses: the first case series in Asia with one-year follow-up. Singapore Med J 2016;57:401-5. 